\title{
Smart Traffic Control Using Sobel Edge Operator
}

\author{
C.V.R.N.Aswin Kumar ${ }^{1}$, Vadlani Dinesh ${ }^{2 *}$ P. Varun Kumar ${ }^{3}$, K.Harika ${ }^{4}$, \\ S.Mounika ${ }^{5}$, K.Sravya Chowdary ${ }^{6}$ \\ ${ }_{1,2,3,4,5,6}$ DST-FIST Sponsored ECE Department, K L University, Vaddeswaram, Guntur district, AP
}

\begin{abstract}
Due to increase in aggregation of vehicles at the road junctions and to avoid accidents at times, there is a need for a smart traffic light controller which can detect vehicle density on the road. This proposal involves image acquisition, vehicle detection and vehicle density through progressive image processing operations applied on the captured images using MATLAB. For multiple road implementations, we use a movable web-camera controlled by a stepper motor and data is stored and further extended for embedded applications. Smart Traffic Control can be achieved by Image acquisition, Edge detection, Image Enhancement techniques and Traffic Regulation.
\end{abstract}

Keywords: Edge detection, Image Enhancement, Vehicle detection.

\section{Introduction}

In the present world where the roads are not even where different classical traffic control techniques were implemented in which magnetic-loop sensors were buried under the road, infra-red and microwave radar sensors fixed to the road-side provide less traffic information and require separate systems for traffic monitoring. Those inductive loop detectors provided a cost-effective solution in the past; however they were subjected to extreme failure rates when installed on rough surfaces and obstruct traffic during operations. These typical infrared sensors get affected by haze and cannot be used for effective surveillance as they don't have durance. It is aware that population of country and number of vehicles is increasing rapidly; hence there is a need of controlling vehicle traffic on the road junctions. This paper aims to depict automatic control system for traffic on busy roads. This technology provides more traffic regulation, combines both surveillance and traffic control technologies, can be easily installed, and is scalable with progress in image processing techniques. This proposal tries to evaluate the process and advantages of the use of image processing algorithms for traffic control. Implementation of this proposal will eliminate the need of traffic personnel at various junctions for regulating traffic. Thus implementation of this technology is worthful for the governance and performance improvement of road traffic.

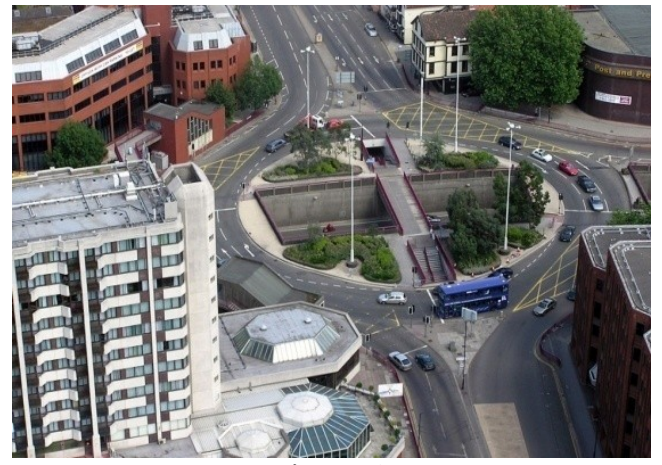

Figure 1

In this approach image capturing of the road traffic is configured with a web cam connected to a stepper controller and thereby each and every road is analyzed and the road traffic density is found. The road with more traffic will be given more preference and given green light and this continues. This can be modeled in MATLAB software and data is sent by serial communication to the microcontroller connected for extended regulation in real-time. 


\section{Block Diagram}

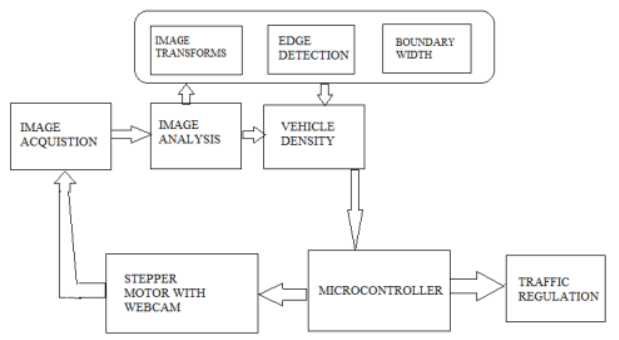

Figure 2

\section{Image Acquistion}

In this system we capture images of the road traffic of 4 roads using a web cam moving with a stepper controller each and every road is analyzed and the road traffic density is found. The traffic density is found by using many image processing algorithms and road vehicle density is found. The road with more traffic will be given more preference and given green light and a timer is given for all the roads depending on the vehicle density obtained.

\section{Image transforms:}

\section{Image Analysis}

There are several image representations in MATLAB where a gray scale image gives a 2-dimensional black and white intensity. The most preferred format for applying image processing operations is grayscale. But when we require color, an RGB color image shall be disintegrated and treated as three disjoint grayscale images. Here grayscale image is converted to binary image for a specific gray threshold of the image. In a binary image the pixels have only two values ' 1 ' for true and ' 0 ' for false.

\section{Edge Detection}

After conversion of image to binary, we have to take care of redundant objects on the road. This alteration of threshold is done by using an efficient edge operator detection algorithm [1]. There are many function specific edge detection techniques where we can apply the image enhancement. Here a Sobel edge operator is proposed to get optimal results for any conditions of the vehicles on the road. A fudge factor of 0.5 is introduced for scaling the threshold to detect the vehicles.

\section{Object Counting by boundaries}

After finding the edges the next stage is to count the number of objects as defined by the edges.

By creating morphological structural elements to define boundaries to the objects and measure the vehicle density in that road. For this we need to dilate the road and then fill the gaps with color so that a correct picture of separate vehicles without connections as a complete one. Hence by finding the length of the boundaries we can find the vehicle density easily. The below figure shows image analysis done on a digitally created traffic for testing this algorithm and this is verified again on a traffic manually tested on a Styrofoam board.

\section{Image Analysis-Input:}

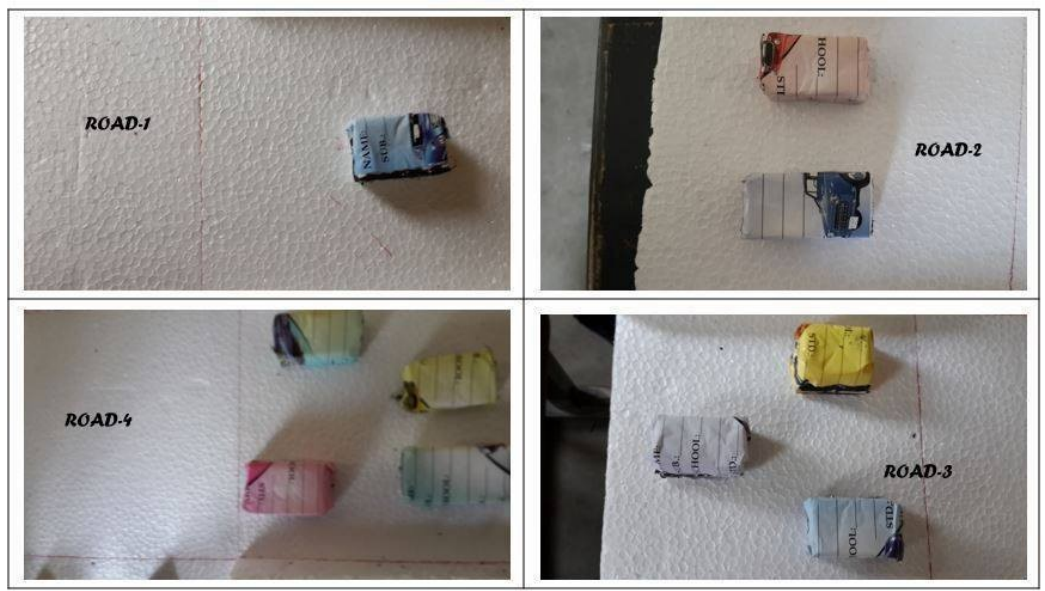

Figure 3 


\section{Results}

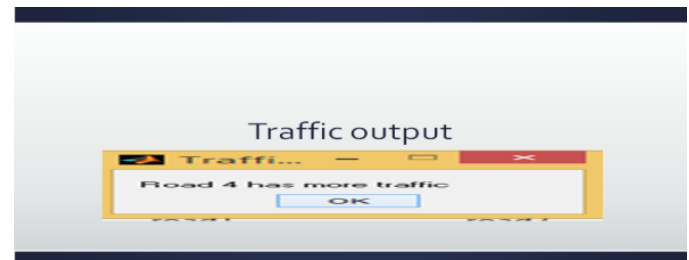

Figure 4

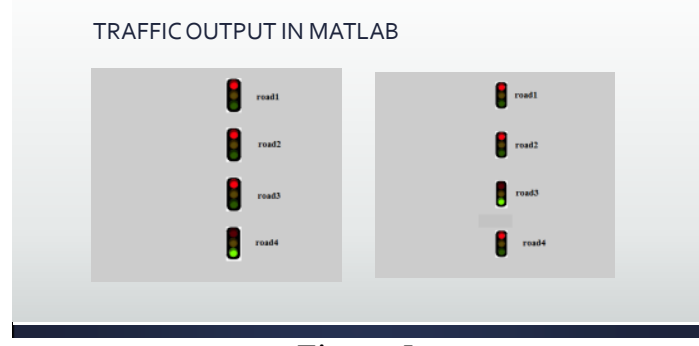

Figure 5

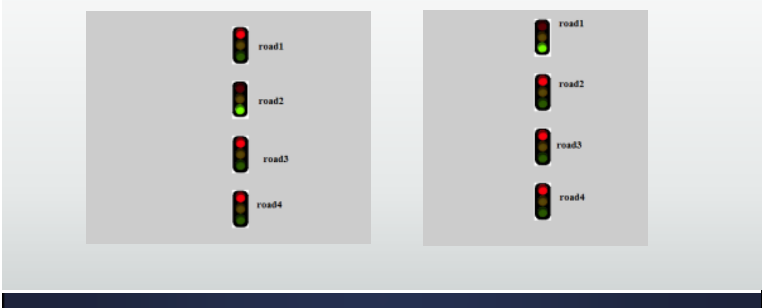

Figure 6

\section{Conclusion}

In this work the images of four roads are taken and by using Sobel Edge Detector, we have considered fudge factor of 0.5 for the Sobel operator and the maximum vehicle density is found for road4 and corresponding lights are given preference and this work is done on software called MATLAB.

\section{Future Work}

This proposed work can be further extended to operate in the busy multiple roads in metropolitan cities where traffic is very high we can implement by using high end DSP boards in real time.

\section{ACKNOWLEDGMENT}

Authors like to express their deep gratitude to Mr.P.Satyanarayana, Assoc. Professor Department of ECE K L University for his great support and encouragement during the implementation of this work and also we would also like to thank our signal processing research members and also our friends for their help in completion of this paper.

[1] Digital image processing by Gonzalez.

\section{References}

[2] Digital Signal Processing By Ramesh Babu.

[3] Intelligent Transportation Systems Video Detection and Monitoring Solutions For Traffic Applications

[4] Promila Sinhmar "Intelligent Traffic Light \& Density Control UsingIr Sensors And Microcontroller".

[5] Pallavi Choudekar "Real Time Traffic Light Control Using Image Processing" 


\section{AUTHOR PROFILE}

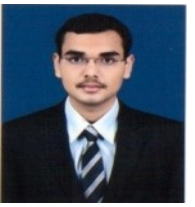

C.V.R.N.Aswin Kumar born in India,A.P in 1994. He is pursuing his B.Tech in Electronics and Comm.Engineering with minor specialization in VLSI from K L University. He is member of IETE. His interest in research includes Antennas and Image Processing. [http://orcid.org/00000003-0349-0984]

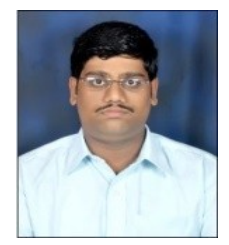

Vadlani.Dinesh was born in India; A.P in 1993. He is pursuing his B.Tech in Electronics \& Communication Engineering from $\mathrm{K} \mathrm{L}$ University. He is getting his Specialization in Networking. He is a member of IETE. He published papers in various International \& National Journals and attended Conferences. His interest in research includes Signal processing and Antennas. At present, he is engaged in Bio-medical Image analysis. [http://orcid.org/00000003-0709-6503].

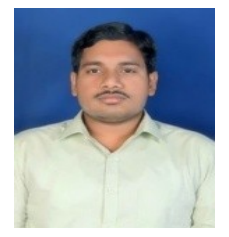

P.Varun Kumar was born in India, A.P, in 1993. He is pursuing his B.Tech in Electrical and Electronics Engineering from K.L. University. He is getting his specialization in energy systems. His research interest includes Energy Systems and image processing.

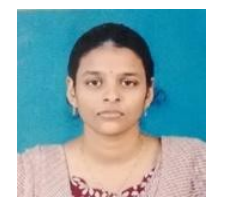

K.Harika was born in India, pursuing her B.Tech in Electronics and Communication Engineering at K L University. She is a member of IETE. She has presented a number of technical papers in Technical symposiums. Her research interests lie in the field of Wireless communications and Antenna design.

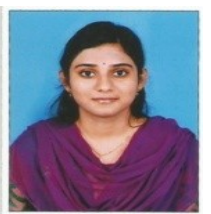

Mounika.Sanikommu was born in India, A.P, in 1994. She is pursuing her B.Tech in Electronics and Communication Engineering from K L University. She is getting her specialization in Networking. She is member of IETE. Her interest in research includes communication systems, antennas and signal processing. At present, she is engaged in patch antenna analysis.

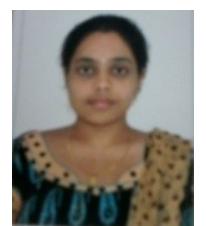

K.SRAVYA CHOWDARY was born in India, A.P, in 1994. She is pursuing her B.Tech in Electronics and Communication Engineering from K L University. She is getting her specialization in communication. She is member of IETE. Her interest in research includes Antennas and Image processing. 